\title{
FACIAL EXPRESSION RECOGNITION BASED ON EDGE DETECTION
}

\author{
Xiaoming CHEN and Wushan CHENG \\ College of Mechanical Engineering, Shanghai University of Engineering Science, \\ Shanghai 201620, China
}

\begin{abstract}
Relational Over the last two decades, the advances in computer vision and pattern recognition power have opened the door to new opportunity of automatic facial expression recognition system[1]. This paper use Canny edge detection method for facial expression recognition. Image color space transformation in the first place and then to identify and locate human face. Next pick up the edge of eyes and mouth's features extraction. Last we judge the facial expressions after compared with the expressions we known in the database. This proposed approach provides full automatic solution of human expressions as well as overcoming facial expressions variation and intensity problems.
\end{abstract}

\section{KEYWORDS}

Facial Expression Recognition; Color Space; Edge Detection.

\section{INTRODUCTION}

Facial expression is a kind of expression of the emotional state, human face has the great power of expression, it provides a powerful and flexible nature expression in communication and emotional state. Using facial expressions can not only express inner emotion, but also provide important clues to the social communication. The facial expression of a speaker accounts for about 55 percent of the effect of the communicated messages, 38 percent of the latter is conveyed by voice intonation and 7 percent by the spoken words. So the facial expression plays an important role in the communication. Research on facial expression recognition system is to detect the real-time facial expression recognition from the information contained in the independent judgment, emotional state and physical needs of human beings and the issue of the correct instruction. Therefore, facial expression recognition system is widely used, such as intensive care room, resistance training, testing etc. However, because the facial expression is complex and changeable, which makes the expression more fuzzy classification and recognize difficulty [2-3].

This paper introduces a study and design of the system of facial expression recognition based edge detection algorithm, first for image preprocessing, image recognition processing allows easy back; and then locate the eyes and lips, individually marked and extract the edge shape feature; finally the system was trained by using face database, achieve the purpose of identifying other face expression. The specific design process is shown in Fig. 1 


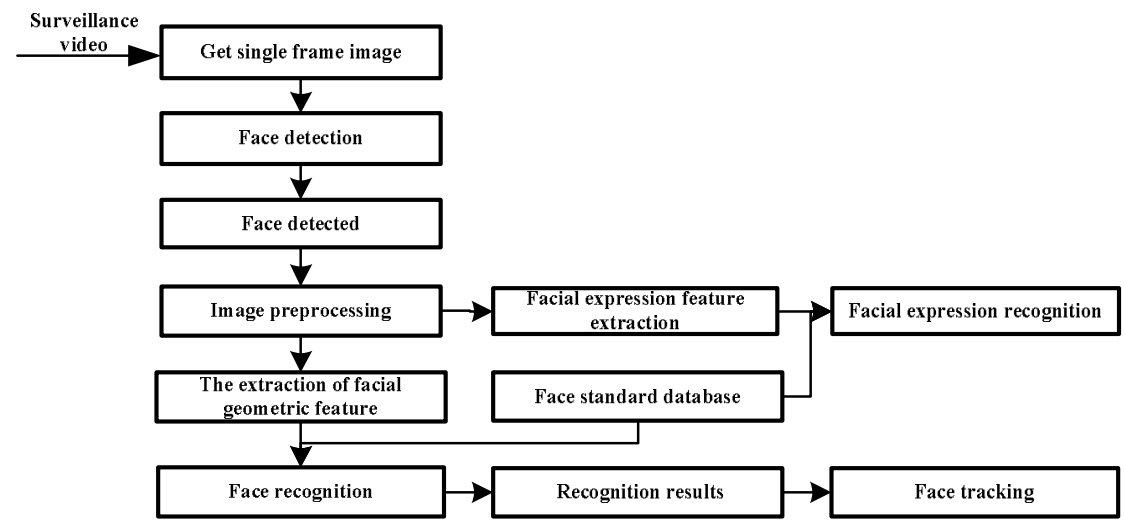

Figure 1. Flow chart of system design

\section{FACE DETECTION AND LOCATION}

Face detection and location is an important premise to extract facial features, therefore, corresponding to the different environment, such as light conditions, face pose, wearing material interference, detection of facial recognition system requirements with high accuracy and robustness.

In the view of the complexity of face detection, the present methods of face detection technology can be broadly divided into two categories: Based on the statistical method and based on the knowledge method [4]. Face location based on statistical method is just as the name suggest, it need a large amount of prior knowledge as theoretical basis, and through kinds of methods such as self learning algorithm of neural network, the subspace dimensionality reduction method and template matching to judge, although these methods are robust, but the large amount of calculation, long computing time, so they are not up to the requirements of real-time identification. Knowledge-based face detection method is to make full use of various characteristic parameters of human face of this unique biological identification as a standard to build models, commonly used has the organ distribution method, color and texture, motion method, symmetric method, etc. Then computer analysis of the images extracted effective characteristic parameters, contrast similarity with known facial feature parameters, so we can realize the face location. For our facial features can be detected by using such as the distance between two eyes, mouth and eyes or eyebrows and eye or some other fixed characteristics; and the color as the main features of the face, the skin color detection can use a variety of color space such as HIS color space, the $\mathrm{YCbCr}$ color space and CIE color space. Research shows that the $\mathrm{YCbCr}$ color space is more suitable for face detection compared to other several color space [5].

We use the camera to photo collection, because the camera photographs in the RGB color space, so it can be through a nonlinear transformation to make photographs by RGB color space to $\mathrm{YCbCr}$ color space.

$$
\left[\begin{array}{c}
Y \\
C b \\
C r
\end{array}\right]=\left[\begin{array}{c}
16 \\
128 \\
128
\end{array}\right]+\left[\begin{array}{ccc}
65.481 & 128.533 & 24.966 \\
-37.797 & -74.203 & 112 \\
112 & -93.786 & -18.214
\end{array}\right]\left[\begin{array}{l}
R \\
G \\
B
\end{array}\right] .
$$




\subsection{The Establishment of Skin Color Model}

Gauss model is a model that decomposed the specific things into a probability density distribution curve of Gauss (The normal distribution curve) to accurately quantify. Because of the similar face skin color distribution and Gauss distribution, so for each point on the face image can use the model of skin color to calculate each point probability. Then establish skin improved grayscale based on skin color probability values of these points, and then the candidate region of human face is the higher probability regions of skin color in the grayscale.

$$
\begin{gathered}
\mathrm{m}=E\{\mathrm{x}\}, \mathrm{x}=(C \mathrm{r}, C \mathrm{~b})^{T}, \\
C=E\left\{(\mathrm{x}-\mathrm{m})(\mathrm{x}-\mathrm{m})^{T}\right\},
\end{gathered}
$$

$m$ is the mean chroma component, $C$ is covariance matrix, Through the Gauss skin distribution calculation of skin probability image of an arbitrary point in value. For example, for a pixel, it is converted from RGB space to YCbCr color space of skin color probability value of the formula is as follows.

$$
p(C r, C b)=\exp \left[-0.5(x-m)^{T} C^{-1}(x-m)\right],
$$

In the formula : $x=(\mathrm{CbCr})^{T}$.

\subsection{Skin Color Segmentation}

Through the Gauss model we know the image of all points on the skin probability value, in order to reduce the effect of high frequency noise, first the pictures will be transferred to the $\mathrm{YCbCr}$ color space from RGB space through the low pass filter, determine the gray level of each pixel point value, and then processing the skin area and other background regions separated threshold segmentation and binaryzation[6], using the following formula to calculate the specific:

$$
f t(x, y)=\left\{\begin{array}{l}
1, \text { if } f(x, y) \geq T \\
0, \text { otherwise }
\end{array}\right.
$$

$T$ is the selected threshold, that is greater than $T$ the pixel is 1 (white),the other is 0 (black). Usually $T$ algorithm has the Bimodal method, $P$ parameter method, Otsu method, iterative method, here we use the Otsu method, the basic idea of Otsu method is to use an assumed gray value $t$ to divided image gray into two groups, when two groups of between class variance is maximum, the gray value of image $t$ is two the value of the optimal threshold [7]. Suppose the image's gray value is $M$, the range is $0 \sim M-1$, select the gray value of $\mathrm{T}$ within this range, the image is divided into two groups of $G 0$ and $G 1, G 0$ contains the pixel gray values in $0 \sim \mathrm{t}, G 1$ 's gray value is in $\mathrm{t}+1 \sim M-1$, with $N$ as pixel number, ni said the number of pixel gray value of $\mathrm{i}$ the number of assumptions, $G 0$ and $G 1$ two groups of pixels which occupies in the whole image respectively, two groups of average gray value, so probability: $\omega_{0}=\sum_{i=0}^{t} p i, \omega_{1}=\sum_{i=t+1}^{M-1} p_{i}=1-\omega_{0}$ .The average gray value are $\mu_{0}=\sum_{i=0}^{t} i p_{i}$ and $\mu_{1}=\sum_{i=t+1}^{M-1} i p_{i} . T$ is the optimal threshold.

$$
\mu=\omega_{0} \times \mu_{0}+\omega_{1} \times \mu_{1}
$$


International Journal of Computer Science \& Engineering Survey (IJCSES) Vol.6, No.2, April 2015

$$
\begin{gathered}
\mathrm{g}(\mathrm{t})=\omega_{0}\left(\mu_{0}-\mu\right)^{2}+\omega_{1}\left(\mu_{1}-\mu\right)^{2}=\omega_{0} \omega_{1}\left(\mu_{0}-\mu_{1}\right)^{2}, \\
T=\operatorname{argmax}(g(t)),
\end{gathered}
$$

Morphological processing: morphology of two value morphology transform image is a view of the process of the collection, its essence is the interaction by the image of objects, or the shape of the set and structural elements of intersection and set operation. The main methods are corrosion $X$ and expansion $Y$ :

$$
\begin{gathered}
X=E \odot \mathrm{B}=\{\mathrm{x}: \mathrm{B}(\mathrm{x}) \subset \mathrm{E}\}, \\
Y=E \oplus B=\{y: B(y) \cap E \neq \Phi\} .
\end{gathered}
$$

Corrosion is a process to eliminate boundary point so that the boundary contraction to interior, it can be used to eliminate small and insignificant objects. Expansion is on the contrary with corrosion, it is a process that all the background point will contact with objects and merged into the object, so that the boundary to the external expansion of the cavity can be used to fill the object.

Through the steps above we can get the effective area of the faces in the image. Fig. 2 shows the process of face detection and location

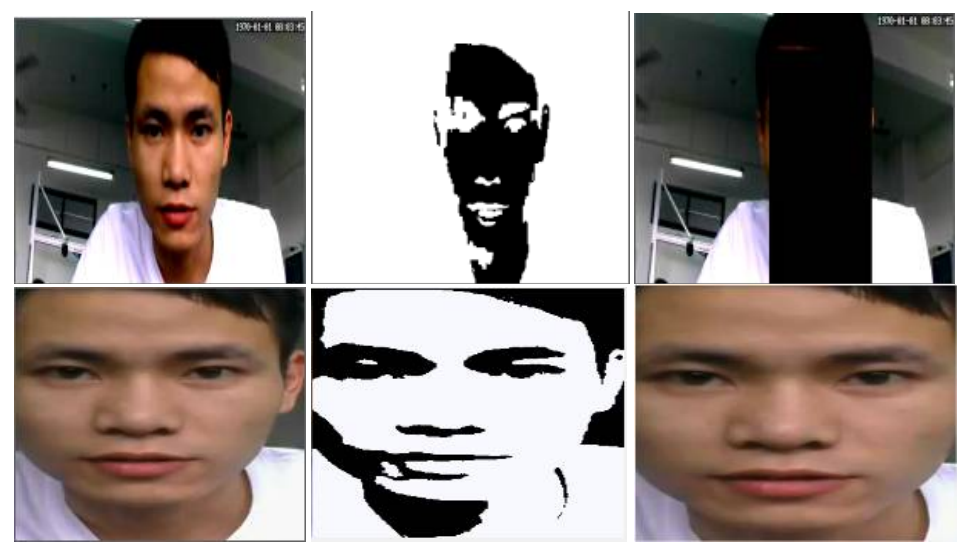

Figure 2.The rough location and precise positioning of human face

\section{Positioning Eyes AND LiPS}

\subsection{Edge Detection}

The edge is the most basic feature of image. The so-called edge is the pixels collection of its surrounding pixels having step edge or changes in the roof edge. Edge exists widely in the object and the background, objects and between element and element. Therefore, it is an important feature of image detection and extraction techniques. The step edge on both sides of the gray value changes obviously, and at the junction of roof edge in gray level increase and decrease. Another is the rise and drop which is formed by the combination of pulse shape edge profile corresponds to a thin strip of gray value mutation. Commonly we use one order and two order derivative to describe and edge detection. 
International Journal of Computer Science \& Engineering Survey (IJCSES) Vol.6, No.2, April 2015

If a pixel in the image of a certain object boundary, then its neighborhood will become a gray level change zone. The two most useful features of this change are the variance rate of gray level and direction, and they express by the magnitude and direction of the gradient vector. Two dimensional function $\mathrm{f}(\mathrm{x}, \mathrm{y})$ is defined as the gradient vector direction derivative.

Edge detection operator checks the neighborhood of each pixel and to quantify the variance rate of gray level, including determines direction, most of the use of methods based on directional derivative mask convolution.

Several commonly used edge detection operator, as shown in Figure 3, the figure can be seen from the results, the Canny operator to detect the edge is the most sophisticated, the most straightforward is Robert operator, Laplace operator and Sobel operator are not obvious.
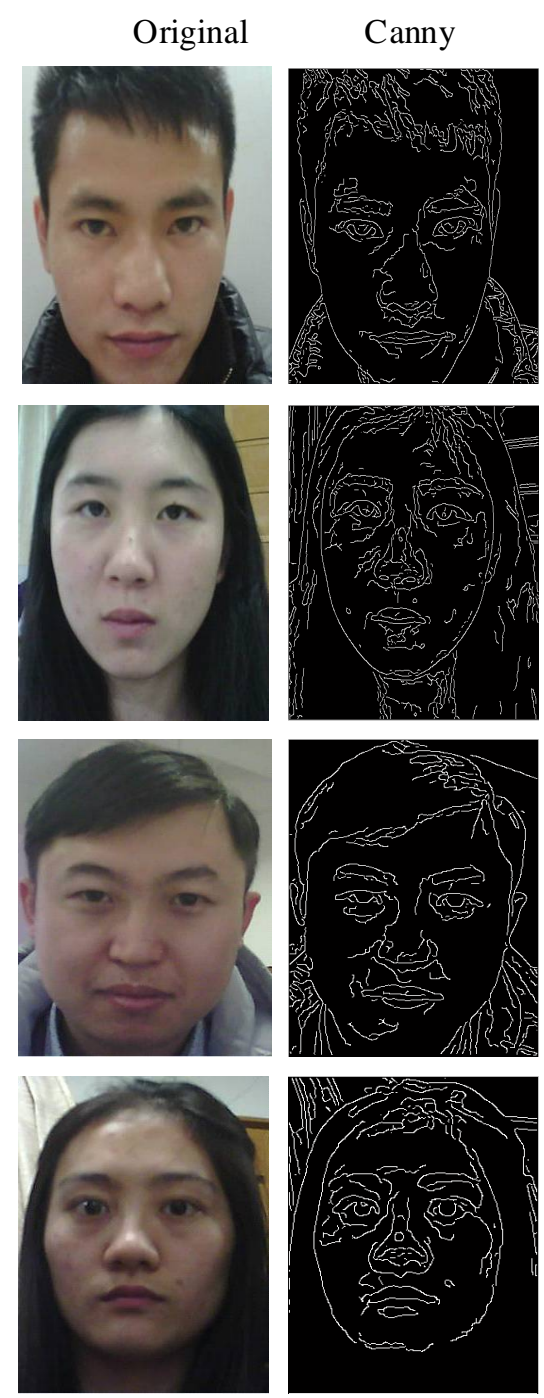
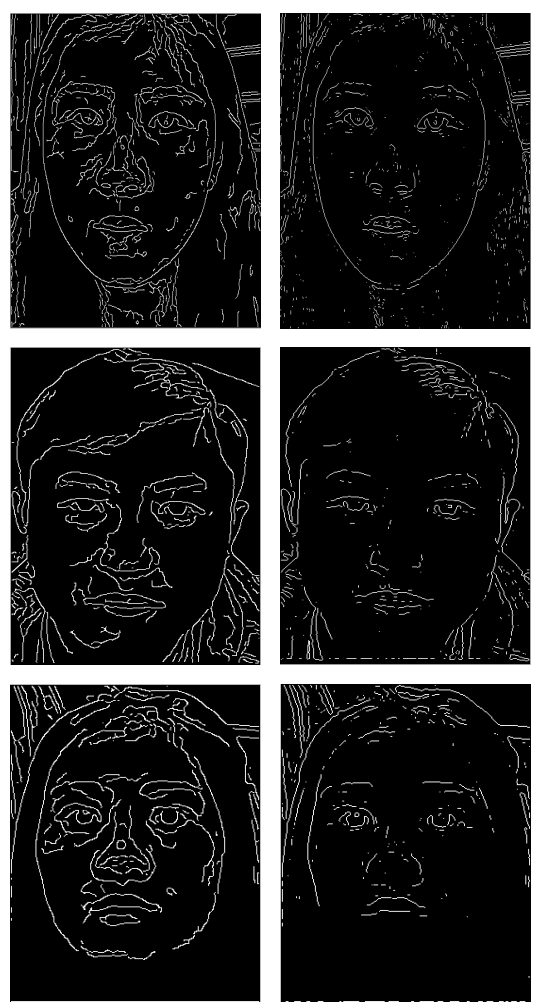

Laplace

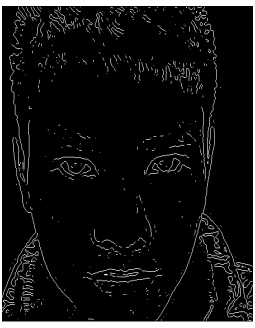

Sobel
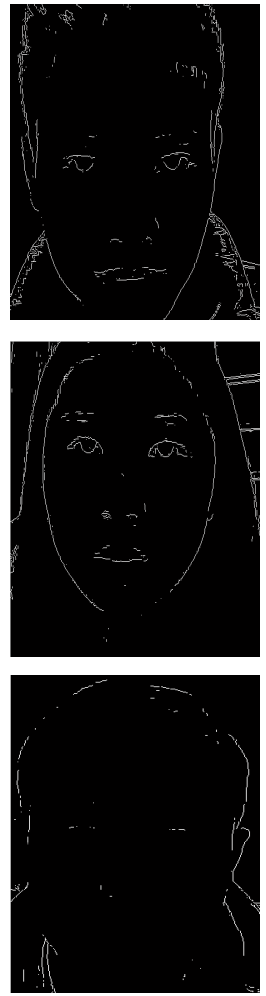

Robert
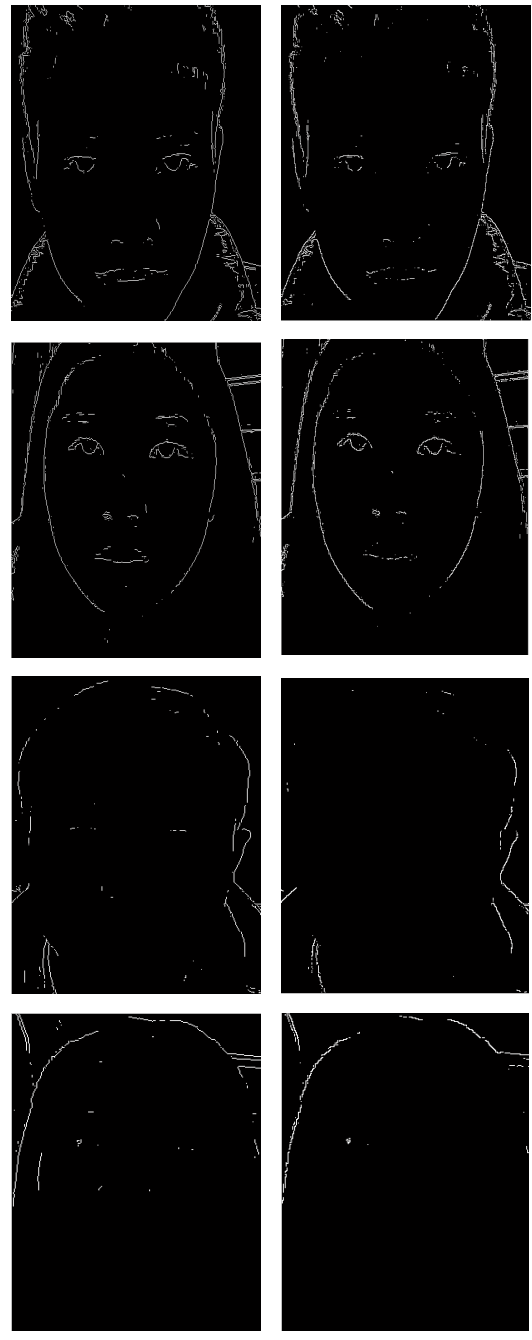

Figure 3 Comparison of the Effect of Edge Detection Algorithm 


\subsection{Canny Edge Detection}

Since the eyes are located in the middle of the top face, lips are in the middle of the bottom face, so the limited area search can improve the detection speed. First estimates the eyes and lips region, and then use the Canny edge detection to determine the exact position of the eyes and lips[8].

(1) Using Gauss filter to smooth the image, The Gauss smoothing function is computed as

$$
\begin{gathered}
H(\mathrm{x}, \mathrm{y})=\mathrm{e}^{-\frac{\mathrm{a}^{2}+\mathrm{b}^{2}}{2 \sigma^{2}}}, \\
G(x, y)=f(x, y) * H(x, y) .
\end{gathered}
$$

(2) To calculate the gradient magnitude and direction by means of finite first-order partial derivative; A first-order differential convolution template:

$$
\begin{gathered}
H_{1}=\left|\begin{array}{cc}
-1 & -1 \\
1 & 1
\end{array}\right|, \\
H_{2}=\left|\begin{array}{cc}
1 & -1 \\
1 & -1
\end{array}\right|, \\
\varphi_{1}(m, n)=f(m, n) * H_{1}(x, y), \\
\varphi_{2}(m, n)=f(m, n) * H_{2}(x, y), \\
\varphi(m, n)=\sqrt{\varphi_{1}^{2}(m, n)+\varphi_{2}^{2}(m, n)} \\
\theta_{\varphi}=\tan ^{-1} \frac{\varphi_{2}(m, n)}{\varphi_{1}(m, n)} .
\end{gathered}
$$

(3) Using gradient direction to suppression non maxima on the gradient magnitude; Fig.4 show the edge direction and angular direction of each point. the purpose is combined with the amplitude and direction of the gradient vector to removal the false edge, specifically to along the gradient direction of eight edge direction of each point one by one to detect modulus maxima, and then compared with the pixels of the partial derivative, then maximum value as the optimal edge points, set the point of gray value as 0 .
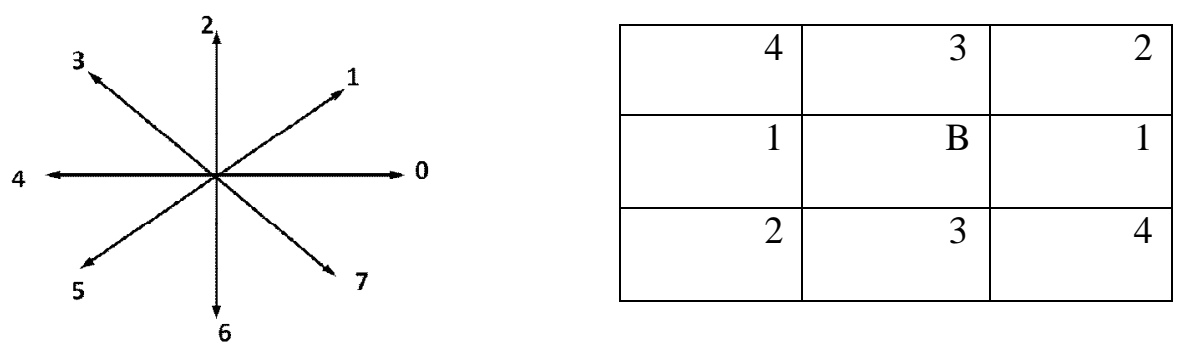

Figure 4. Schematic diagram of the edge direction and 8 neighborhood angular direction 
(4) Using double threshold algorithm connecting edge; through suppression non maxima we can removed the false edge beside real, for the false edge beside false edge need to further removed to draw the edge contour clearly. The most important things are the two adaptive threshold $\tau_{1}$ and $\tau_{2}$, and general requirements $2 \tau_{1} \approx \tau_{2}$, and the strong threshold $\tau_{2}$ determined true edge image $\mathrm{N} 2[\mathrm{i}, \mathrm{j}]$, then the weak threshold on the true edge connection processing, making sure the edge is smoothly and completely [9].

According to different profile of the two eyes, testing individually and extract eyes and lips edges, the Fig. 5 shows four kinds of facial expression by using the extracted edge algorithm

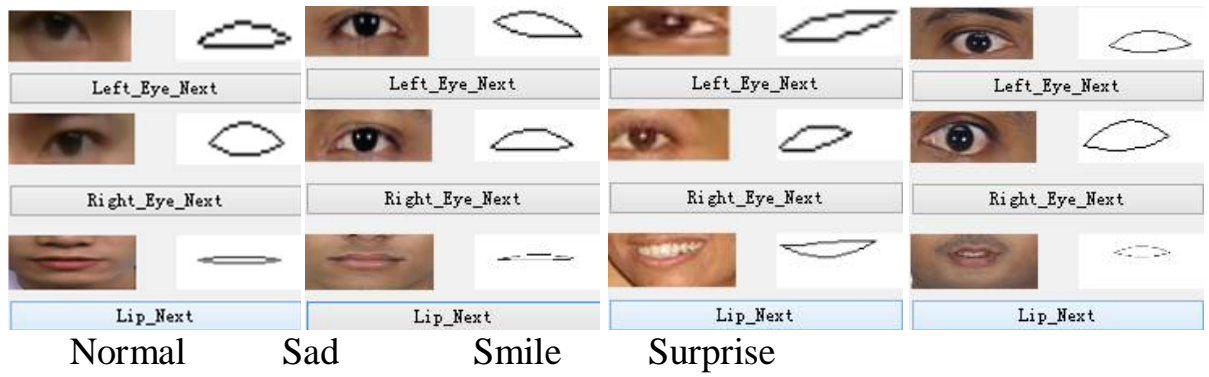

Figure 5.Four kinds of facial expression feature extraction

\section{EXPERIMENTAL RESUlts}

In order to test the accuracy of the recognition method, we choose the suitable for the color of skin of Japanese Jaffe facial expression database as the material, and carries on the classification according to the requirements of the experiment, selecting four kinds of facial expression, each expression selects three pictures, including two as the training group, the other as a test group [10]. Then use Matlab to training the selected samples, the training results as shown in Table 1

Table 1.Test results

\begin{tabular}{|c|c|c|c|c|}
\hline $\begin{array}{c}\text { Facial Expression } \\
\text { Discrimination (\%) }\end{array}$ & Normal & Sad & Smile & Surprise \\
\hline Canny & 100 & 91.3 & 99.4 & 95.7 \\
\hline Laplace & 93.2 & 87.1 & 89.5 & 85.6 \\
\hline Sobel & 63.4 & 49.7 & 66.8 & 64.9 \\
\hline Robert & 49.8 & 30.1 & 42.5 & 48.2 \\
\hline
\end{tabular}

As can be shown in the table, the algorithm of edge detection for normal and smile recognition can achieve high accurate precision, which indicates that the algorithm of facial expression recognition rules is effectively for normal and smile; as sorrow and surprise, because those kinds of expression the physiological performances are different between different people, although the algorithm rules cannot achieve without error identification, it also has high discrimination. In addition, the other three kinds of edge detection operator are tested, by contrast, the recognition effect is obvious worse than the first. 
International Journal of Computer Science \& Engineering Survey (IJCSES) Vol.6, No.2, April 2015

Laplace operation use only one template, so the amount of calculation is small. Laplace algorithm is sensitive to the noise in the image, but usually produces two pixels wide edge, so rarely used for edge detection, is generally used to determine the pixel in the image is dark or light over there in the known edge pixels after.

Using Sobel edge detection operator is the point $(\mathrm{x}, \mathrm{y})$ approximation of the gradient amplitude. Sobel edge detection operator to better edge effect at the same time, and could smooth noise, reduces the sensitivity to noise. But the detection of Sobel edge detection operator is thick, also can detect some false edge, so the edge detection accuracy is relatively low.

The Robert edge detection operator is the most simple, but there are some limitations in its function. Because the Robert edge detection operator is the difference of adjacent pixels detection using two diagonal direction of the image gradient magnitude of the difference is in the approximate locations in the gradient magnitude values. Detecting horizontal and vertical edges better, but in the tilt direction of the edge detection accuracy is relatively poor, but also more sensitive to noise. But its advantages are simple and fast.

In this experiment, the edge detection is the basis and premise of facial expression recognition, The clear outline for detection of eyes and mouth parts are directly related to the accuracy of face recognition, which requires the operator has good anti noise ability, also should have a better edge detection precision in each direction, obviously Canny operator is the best choice.

\section{CONCLUSIONS}

In this paper, we introduced the design of a facial expression recognition system based on edge character, is the main characteristics and training database under the condition of different expression of eyes and lips edge by face in the ratio, thus the face recognition. The design of other recognition system has a very high robustness with respect to conventional face recognition effect, fast and accuracy. From the final recognition effect, that the gradient operator edge algorithm is sensitive to noise, so it is suitable for the image edge gray value in the image is sharp and under the condition of litter noise. However, the method used in this paper for photo quality (such as light, high requirements definition), another paper training and test images are not considering the effect of face wear fabrics, this also will be the direction of future research.

\section{ACKNOWLEDGEMENTS}

This work is supported by the Shanghai Science and Technology Committee.

\section{REFERENCES}

[1] Balasubramani, A.; Kalaivanan, K.; Karpagalakshmi, R.C.; Monikandan, R., "Automatic facial expression recognition system," Computing, Communication and Networking, 2008. ICCCn 2008. International Conference on , vol., no., pp.1,5, 18-20 Dec. 2008

[2] Thomas, N.; Mathew, M., "Facial expression recognition system using neural network and MATLAB," Computing, Communication and Applications (ICCCA), 2012 International Conference on , vol., no., pp.1,5, 22-24 Feb. 2012

[3] Girard, J.M.; Cohn, J.F.; Mahoor, M.H.; Mavadati, S.; Rosenwald, D.P., "Social risk and depression: 
International Journal of Computer Science \& Engineering Survey (IJCSES) Vol.6, No.2, April 2015

Evidence from manual and automatic facial expression analysis," Automatic Face and Gesture Recognition (FG), 2013 10th IEEE International Conference and Workshops on , vol., no., pp.1,8, 2226 April 2013

[4] Zhi-Huang Zou, Wu-Shan Cheng, Xin Sun," real time face recognition system based on DSP", modern machinery. [J]. 2008,02:64-66.

[5] Hai-Yang Wang," The research on drowsy detection based on the information of eye images", Shenyang University of Technology, 2007

[6] Jiang-Jiang Huang, Research on driver fatigue test measurement system based on identification of human eye.[D]. Jilin University, 2010

[7] Yong Cao, The research of face recognition algorithm based on geometric features. [D]. Jilin University, 2008

[8] Jun Zeng, Research on image edge detection and its application. [D]. Huazhong University of Science and Technology, 2011

[9] Na Wang,Xia Li,A new improve edge detection algorithm based on the Canny operator. [J]. Journal of Shenzhen University, 2005,02:149-153.

[10] Rong-Ping Dong,Bo-Ling Tang, Facial Expression Recognition Using DCT-Back Propagation Neural Network. [J]. microcomputer information, 2005,18:142-144.

\section{Author}

Xiaoming CHEN was born in 1991,and now the Shanghai University of Engineering Science postgraduate. His present research interest is image analysis and processing.

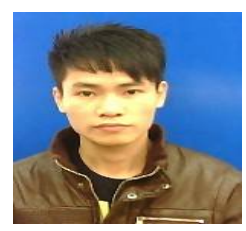

